\title{
Previously unseen brain-eyelid thermal tunnel reveals biological waveguide and transorbital thermophysical pathway to the brain
}

\author{
M Marc Abreu ${ }^{1,2}$, Ricardo L Smith ${ }^{3}$, Keith Ruskin ${ }^{1,4}$, Adriano F Da Silva ${ }^{5}$, Ala S \\ Haddadin $^{1}$, Michael F Bergeron ${ }^{6}$, Trevor M Banack ${ }^{1}$, and David G Silverman ${ }^{1,7}$ \\ ${ }^{1}$ Department of Anesthesiology, Yale University School of Medicine \\ ${ }^{2}$ Department of Ophthalmology and Visual Science, Yale University School of Medicine \\ ${ }^{3}$ Department of Morphology and Genetics, Paulista School of Medicine, Federal University \\ of São Paulo \\ ${ }^{4}$ Department of Neurosurgery, Yale University School of Medicine \\ ${ }^{5}$ Department of Radiology, Heart Institute, University of Sao Paulo Medical School \\ ${ }^{6}$ Department of Physical Therapy, Medical College of Georgia, Augusta University \\ ${ }^{7}$ John B Pierce Foundation Laboratory, Yale University
}

October 9, 2020

\begin{abstract}
Fat is recognized as low thermoconductivity $(k)$ tissue and barrier for thermal transmission. We here identify and characterize morphologically, radiologically, and thermo-physically fat-enabled bilateral integrated blood/fat/skin thermal pathways, referred as Brain-eyelid Thermal Tunnels (BTT), that enable undisturbed thermal transmission between brain and uniquely radiant high- $k$ skin in the right and left eyelids. We elucidated cranial "aberrancies", justified the facial "death triangle" and uncovered perihypothalamic thermoregulatory-sensory triunal inherent to brain thermal homeostasis that revealed thermal communication among brain, heart, and environment. Our results introduce the first evidence for a brain-surface thermo-physical pathway and biological thermal waveguide. Said transmission was documented by an isolated beam of emission at the ipsilateral BTT terminus. We showed brain thermal transfer via BTT during neurosurgery by comparison of noninvasive temperature measurement at the $\mathrm{BTT}$ site $\left(\mathrm{BTT}^{\circ}\right)$ to direct temperature measurement in brain parenchyma $\left(\right.$ Parenchyma $\left.^{\circ}\right)$ in open craniotomy $\left(\mathrm{BTT}^{\circ}\right.$ - Parenchyma ${ }^{\circ}=0.012 \pm 0.2^{\circ} \mathrm{C}, \mathrm{p}=0.91$ ), as opposed to $0.45^{\circ} \mathrm{C}$ difference between $\mathrm{BTT}^{\circ}$ and core temperature. This indicates that $\mathrm{BTT}^{\circ}$ provides an accurate noninvasive method for measuring human brain temperature unimpeded by the body's thermal barrier through transorbital thermo-physical configurations.
\end{abstract}

\section{Hosted file}

Authorea_BrainThermophysicalTunnel_TLY_Abreu et al_Yale Univ_0ct0820-Submitted.pdf available at https://authorea.com/users/364278/articles/485634-previously-unseen-brain-eyelidthermal-tunnel-reveals-biological-waveguide-and-transorbital-thermophysical-pathway-tothe-brain 